Disclosure of Interest: None declared

DOI: 10.1136/annrheumdis-2017-eular.6765

\section{FRI0742-HPR DOES THE USE OF TECHNOLOGICAL DEVICES IMPROVE THE RELIABILITY OF MEASURING THE ACTIVE CERVICAL RANGE OF MOTION IN PATIENTS WITH NECK PAIN? A SYSTEMATIC REVIEW WITH META-REGRESSION}

A. Rondoni ${ }^{1}$, G. Rossettini ${ }^{1}$, M. Strobe ${ }^{1}$, F. Gallo ${ }^{2}$, D. Ristori ${ }^{1}$, M. Testa $^{1}{ }^{1}$ ${ }^{1}$ Department of Neuroscience, Rehabilitation, Ophthalmology, Genetics, Maternal and Child Health, University of Genova, Savona; ${ }^{2}$ Department of Health Sciences, University of Genova, Genova, Italy

Background: Economic impact of neck pain shows an increasing trend. In order to limit the costs of spine disorders management, it is important to assess clinical efficacy and the cost-worthiness of new technological devices, recently introduced in physical therapist's clinical practice

Objectives: This systematic review compares, in patients with non-specific neck pain, the reliability of measures of Active Cervical Range of Motion (ACROM) detected with technological devices with those assessed with low cost, commonuse devices. As secondary outcomes, it was investigated if ACROM reliability depends on the plane on which the measured movement is performed

Methods: The literature search was carried out in Medline, Scopus, Embase, The Cochrane Library, CINHAL, PEDro and grey literature until August 2016. Inclusion criteria were: reliability design, population of adults with nonspecific neck pain, examiners of any level of experience, measures repeated at least twice and statistics indexes on reliability. Exclusion criteria were: other study designs, asymptomatic population or mixed population, single or none ACROM measure, inadequate statistics. The risk of bias was assessed by QAREL. It was considered inexpensive a device that costs at maximum 500 euros. A Univariate, and a Multivariate Analysis, were performed by using the Linear Mixed-Effect Model Results: Searching the databases yielded 35,151 records. Nine studies met all eligibility criteria. The QAREL mean score of the selected studies was 3.7 out of 11. No significant effect of the type of device (inexpensive versus expensive) on ICC was observed for intra-rater (ICC $=0.93 v s 0.91 \mathrm{p}$-value $>0.99$ ) and inter-rater reliability $($ ICC $=0.80 \mathrm{vs} 0.87 \mathrm{p}$-value $>0.99)$ [Table 1]. The plane of movement did not affect inter-rater reliability ( $p$-value $=0.11$ ) while significantly influenced the intra-rater reliability $(p$-value $=0.0001)$ assessed with low-cost devices. Intra-rater reliability significantly decreases $(p$-value $=0.0129)$ in frontal plane movements (side bending) compared with movement on the sagittal plane (flexion-extension).

Table 1. Comparison of intra and inter-rater reliability between tool types

\begin{tabular}{lccc}
\hline Characteristics & ICC Descriptive statistics & \multicolumn{2}{c}{ Mixed effect model } \\
\cline { 3 - 4 } & Mean (SD) & $\beta(95 \% \mathrm{Cl})$ & $\mathrm{p}$-value \\
\hline $\begin{array}{l}\text { Intra-rater reliability } \\
\text { Tool Type }\end{array}$ & & 0.99 \\
$\quad$ Expensive & $0.91(0.07)$ & $0.01(-0.04: 0.06)$ & \\
$\quad$ Inexpensive & $0.93(0.02)$ & 0 & 0.56 \\
$\quad$ Direction & & $0.03(-0.01: 0.07)$ & \\
$\quad$ Flexion \& Extension & $0.91(0.07)$ & $0.01(-0.02: 0.04)$ & \\
$\quad$ Rotation & $0.95(0.02)$ & $0.00(-0.01: 0.01)$ & 0.79 \\
$\quad$ Side Bending & $0.92(0.01)$ & & 0.99 \\
Age & rho $=0.29$ & 0 & \\
Inter-rater reliability & & $0.03(-0.15: 0.21)$ & \\
Tool Type & & 0 & 0.06 \\
$\quad$ Expensive & $0.87(0.09)$ & $0.04(-0.02: 0.11)$ & \\
$\quad$ Inexpensive & $0.80(0.12)$ & $-0.04(-0.10: 0.01)$ & \\
Direction & & & \\
$\quad$ Flexion \& Extension & $0.82(0.09)$ & $0.01(-0.01: 0.03)$ & 0.43 \\
$\quad$ Rotation & $0.86(0.11)$ & & \\
$\quad$ Side Bending & $0.77(0.13)$ & & \\
Age & rho $=0.58$ & & \\
\hline
\end{tabular}

Conclusions: The use of expensive devices to measure ACROM in adults with nonspecific neck pain does not seem to improve the reliability of the assessment. The assessment of side bending showed the lowest level of inter-raters reliability. Since the quality of the analysed studies is low, the conclusion of the present study should be taken cautiously

Disclosure of Interest: None declared

DOI: 10.1136/annrheumdis-2017-eular.3991

\section{FRI0743-HPR THE ASSOCIATION OF PHYSICAL FITNESS COMPONENTS WITH SLEEP QUALITY IN WOMEN WITH FIBROMYALGIA: THE AL-ÁNDALUS PROJECT}

M. Borges-Cosic ${ }^{1}$, V. Segura-Jiménez ${ }^{2}$, F. Estévez-López ${ }^{1,3}$,

I.C. Álvarez-Gallardo ${ }^{2}$, V.A. Aparicio ${ }^{4}$, P. Acosta-Manzano ${ }^{1}$,

B. Gavilán-Carrera ${ }^{1}$, M. Delgado-Fernández ${ }^{1}$. ${ }^{1}$ Physical education and sport,

University of Granada, Granada; ${ }^{2}$ Physical Education, University of Cádiz, Cádiz,

Spain; ${ }^{3}$ Department of Psychology, University of Utrecht, Utrecht, Netherlands;

${ }^{4}$ Department of Physiology, University of Granada, Granada, Spain

Background: Fibromyalgia women report poorer sleep quality, fewer hours of sleep, greater nighttime awakenings, and non-restorative sleep compared with healthy people and other clinical populations (Diaz-Piedra et al., 2015). Previous literature has shown a positive effect of exercise training on sleep quality in women with fibromyalgia (Munguía-Izquierdo et al., 2008). Given the close relationship between exercising and physical fitness improvement, it is important to ascertain the individual influence of physical fitness components on sleep quality in this population.

Objectives: To examine the individual association of physical fitness components with sleep quality in women with fibromyalgia.

Methods: A total of 444 women with fibromyalgia (means $\pm s d=51.3 \pm 7.5$ years old) from Andalusia (southern Spain) were included in this cross-sectional study. The Senior Fitness test battery was used to assess physical fitness. This battery consists of the chair sit-and-reach, back scratch, 30-second chair stand, arm curl, 8-foot up-and-go (lower score indicates better performance) and 6-minute walk tests, which assess lower body flexibility, upper body flexibility, lower body strength, upper body strength, speed agility and cardiorespiratory fitness, respectively. Sleep quality was measured using the total score of the Pittsburgh Sleep Quality Index (PSQI) questionnaire (greater score indicates worse sleep quality). Linear regression was used to explore the association of each physical fitness component with global sleep quality. Analyses were controlled for age, total body fat percentage, marital status, educational level, medication for relaxation/sleep and regular menstruation.

Results: Lower body flexibility $(b=-0.033, \beta=-0.103)$, upper body flexibility $(b=-$ $0.031, \beta=-0.096)$, lower body strength $(b=-0.191, \beta=-0.157)$, upper body strength $(b=-0.111, \beta=-0.142)$ and cardiorespiratory fitness $(b=-0.008, \beta=-0.154)$ were inversely associated with greater scores in the PSQI questionnaire (all, $p<0.05$ ). Speed-agility $(b=0.282, \beta=0.128)$ was positively associated with the score in the PSQI $(p<0.01)$

Conclusions: The current study showed that higher physical fitness is generally associated with better sleep quality in women with fibromyalgia. It is important to mention that all the physical fitness components seem to be associated with sleep quality. Lower body strength and aerobic capacity were the components that showed the strongest associations with sleep quality. Future studies are warranted to elucidate the causality of these relationships.

References:

[1] Diaz-Piedra, Carolina et al. 2015. Sleep Disturbances of Adult Women Suffering from Fibromyalgia: Asystematic Review of Observational Studies. Sleep Medicine Reviews 21:86-99.

[2] Munguía-Izquierdo, D. \& Legaz-Arrese, A. Assessment of the effects of aquatic therapy on global symptomatology in patients with fibromialgia síndrome: a randomized controlled trial. Arch. Phys. Med. Rehabil. 2008, 89:2250-2257.

Acknowledgements: This study was supported by the Spanish Ministry of Economy and Competitiveness (I+D+I DEP2010-15639; DEP2013-40908-R).

Disclosure of Interest: None declared

DOI: 10.1136/annrheumdis-2017-eular.4801

\section{FRI0744-HPR THE EFFECTIVENESS OF THE LOWER DOSE OF LASER TREATMENT ON KNEE OSTEOARTHRITIS}

E. Ekiz ${ }^{1}$, K. Capaci ${ }^{2}$, M. Korkmaz ${ }^{3} .{ }^{1}$ Physical therapy and rehabilitation, Alaşehir State Hospital, Manisa; ${ }^{2}$ Physical therapy and rehabilitation, Ege University, Izmir; ${ }^{3}$ Physical therapy and rehabilitation, Afyonkarahisar State Hospital, Afyonkarahisar, Turkey

Background: During knee osteoarthritis (OA) therapy; medications, physical therapy modalities, exercises and surgical procedures are used. In previous studies, the benefits of low level laser therapy (LLLT) for pain in the low back, shoulder, elbow, and hand have reported.

Objectives: In this study we aimed to investigate the effectiveness of LLLT on pain, functional status, and quality of life in patients with knee osteoarthritis.

Methods: Patients with Kellgren-Lawrence stage 2-3 knee osteoarthritis were enrolled. It was planned as a prospective, randomized and double-blind study. Group 1 received active laser therapy and group 2 placebo laser therapy. Patients have been provided active/placebo laser therapy for two weeks ( 5 days a week, a total of 10 sessions). Patients were evaluated before, immediately after and 1 or 3 months after treatment. Outcome measurements included pain intensity at rest and at movement on visual analog scale, knee function using Western Ontario McMaster Universities Osteoarthritis Index (WOMAC) scale, active/passive joint range of movement, quality of life using Short form 36 (SF-36), and 15-meter walking distance, painless walking distance.

Results: In this study, we observed improvements in knee pain, 15-meter walking distance, WOMAC pain, stiffness and function scores, Lequesne index, and SF-36 physical function and social function $(p<0.05)$ in active LLLT group. Also, significant improvements were detected in SF-36 physical role, mental health, vitality and emotional role parameters compared with placebo laser group $(p>0.05)$.

Conclusions: The present study demonstrated that LLLT is safe and effective upon pain and functional parameters on knee osteoarthritis. However, we think further long-term studies which will include more patients in order to determine the priority and effectiviness are required.

Disclosure of Interest: None declared

DOI: 10.1136/annrheumdis-2017-eular.5187 